\title{
Attentional requirements of postural control in people with spinal cord injury: the effect of dual task
}

\author{
CM Tse ${ }^{1,2}$, MG Carpenter $^{1,2}$, T Liu-Ambrose ${ }^{3}$, AE Chisholm ${ }^{1,2}$ and T Lam ${ }^{1,2}$
}

Study Design: Cross-sectional study.

Objectives: To investigate the attentional requirements for maintaining standing balance in people with spinal cord injury (SCI) using a dual-task paradigm and to compare standing balance performance between SCl and able-bodied (AB) controls.

Setting: Laboratory

Methods: Nine adults with incomplete $\mathrm{SCl}$, who were able to stand unassisted were recruited, along with eight $\mathrm{AB}$ controls. Subjects performed a dual task involving counting backwards by $3 \mathrm{~s}$ out loud while standing with eyes open or closed. The primary outcome measures were the differences between $\mathrm{SCl}$ and control groups for movement reinvestment and the change in performance between single task and dual task for: (i) maximum standing time (STime); (ii) error ratio and total number of words uttered; and (iii) center of pressure measures. Perceptual measures included perceived mental workload, fear and confidence.

Results: SCl subjects stood for shorter duration during dual task (stand and count) than single task (stand) compared with controls during eyes closed. Significant differences between groups were observed for movement reinvestment, center of pressure, perceived mental effort, fear and confidence. No significant effects were observed for math-task performance.

Conclusions: Total STime during eyes closed is adversely affected by the addition of a math task for SCl subjects. Perceptual measures appear to correspond to increases in postural sway and conscious control of standing in subjects with SCl. Individuals who can stand for $>60 \mathrm{~s}$ with eyes closed do not appear to be significantly affected by the addition of a concurrent secondary task of minimal mental workload.

Spinal Cord (2017) 55, 915-920; doi:10.1038/sc.2017.42; published online 16 May 2017

\section{INTRODUCTION}

Postural control in sitting or standing is essential for performing the functional activities that underpin all activities of daily living. Standing has been traditionally considered an unconscious, automatic task, relying on feedback control from somatosensory and visual input, while attentional requirements are minimal. ${ }^{1}$ However, evidence from dual-task paradigms, where postural and suprapostural (secondary) activities are performed concurrently suggest that there are significant attentional requirements in postural control. ${ }^{2,3}$

Dual task studies have revealed that increased dependency on attentional processes for postural control seems more apparent with changes in the central nervous system, such as with aging and disease $^{2,4-8}$ in people with spinal cord injury (SCI), where information to and from the brain has been disrupted, weakness, spasticity, sensory deficits and/or pain can impact balance. ${ }^{9}$ Indeed, unsteadiness during standing is exacerbated in conditions where somatosensory or visual challenges are presented. ${ }^{9,10}$ Postural control is thought to become more attentionally demanding as the postural environment is made more challenging and when a secondary task is required. ${ }^{11}$ Under these dual-task conditions, there may be more interference or competition between processes involved in cognition and postural control, leading to a disruption of the automaticity of movements (as in quiet standing) to a more conscious form of movement control. ${ }^{12}$ Such a shift from an automatic, unconscious level of control to one relying more on conscious control is termed reinvestment ${ }^{12}$ and may have detrimental effects on function. ${ }^{13}$

Populations with decreased balance ability due to deficits in either their somatosensory, visual or vestibular systems compensate by allocating greater cognitive resources to remaining systems to maintain postural stability. ${ }^{5}$ Such reallocation of attentional resources to an apparently simple task, such as quiet standing, that forms the basis of many daily tasks has implications for understanding functional outcomes following an SCI. Thus, the overall objective of this study was to explore the effects of attentional requirements of postural control in people with SCI by comparing standing time (STime), movement reinvestment, postural sway and math-task performance during standing between SCI and able-bodied (AB) controls in a dual-task paradigm. We chose a mathematical task which has been shown to effectively degrade postural stability in healthy older and young adults. ${ }^{11,14-16}$

\footnotetext{
${ }^{1}$ School of Kinesiology, University of British Columbia, Vancouver, British Columbia, Canada; ${ }^{2}$ International Collaboration on Repair Discoveries (ICORD), Vancouver Coastal Health Research Institute, University of British Columbia, Blusson Spinal Cord Center, Vancouver, British Columbia, Canada and ${ }^{3}$ Department of Physical Therapy, Faculty of Medicine, University of British Columbia, Vancouver, British Columbia, Canada

Correspondence: Dr T Lam, International Collaboration on Repair Discoveries (ICORD), Vancouver Coastal Health Research Institute, University of British Columbia, Blusson Spinal Cord Center, 818 West 10th Avenue, Vancouver, British Columbia, Canada V5Z 1M9.

E-mail: tania.lam@ubc.ca

Received 27 November 2016; revised 25 March 2017; accepted 28 March 2017; published online 16 May 2017
} 


\section{MATERIALS AND METHODS}

\section{Participants}

Adult subjects with motor-incomplete SCI and age- and sex-matched $\mathrm{AB}$ controls were recruited. Inclusion criteria were: being medically stable and able to stand for at least $30 \mathrm{~s}$ unassisted without the use of hand-held aids, though the use of orthoses was permitted. Exclusion criteria were: presence of cognitive or visual impairments, inability to follow instructions, orthopedic or neurological problems (other than SCI) or cardiovascular dysregulation (for example, orthostatic hypotension) that could interfere with postural control. All procedures were approved by the university research ethics board and all participants provided written informed consent before any experimental procedures. We certify that all applicable institutional and governmental regulations concerning the ethical use of human volunteers were followed during the course of this research.

\section{Postural conditions}

Three 2-min trials of two postural conditions (sitting and standing) were performed. For the sitting condition, subjects sat supported in a chair, eyes focused on a target $2 \mathrm{~m}$ in front with hands in their lap. For the standing condition, subjects were asked to stand barefoot (unless wearing orthoses, where shoes were permitted) between parallel bars in their preferred position on two Bertec force platforms (Bertec FP 4550, Bertec Corporation, Columbus, $\mathrm{OH}$, USA), looking straight ahead at a target $2 \mathrm{~m}$ away and with their arms by their sides. Analog signals from the force plates were streamed to a data acquisition system at $100 \mathrm{~Hz}$ using custom-written software in Labview 8.6 (National Instruments, Austin, TX, USA). Subjects' performance was videotaped for offline analysis to measure the STime. For all standing trials, the subject was asked to focus on balance. To ensure consistent foot placement between trials, outlines of the feet were traced on the force platform. Commencement of data collection occurred $\sim 10-20 \mathrm{~s}$ after achieving standing balance in order to account for transient effects seen in the first $20 \mathrm{~s}$ of quiet stance. ${ }^{17}$

\section{Suprapostural task and vision conditions}

Single-task and dual-task performance were assessed under two vision conditions: eyes open and eyes closed (Table 1). For the dual-task conditions, subjects were asked to count out loud and backwards by $3 \mathrm{~s}$ starting from a random number provided by the researcher. Data were collected until the subject opened their eyes (in the eyes closed condition) or until their hands left their sides or touched the parallel bars. Rest intervals of $10 \mathrm{~min}$ were provided between trials to decrease the effects of fatigue while providing the time to complete the perceptual and functional measures. The order of the trials was randomized.

\section{Outcome measures}

Our primary measures were STime, math-task performance (error ratio (ER) and total words uttered), movement reinvestment and postural control. Our secondary measures were subjective mental workload, fear and confidence.

STime was defined by the start of the trial until the subject was seen to lose their balance or open their eyes for both single- and dual-task conditions.

ERs were calculated as the ratio of the number of incorrect responses to the total number of words uttered (TOT) including any corrections made. ${ }^{16}$ Average ER and TOT were calculated for each subject and then averaged across all participants. Note that for the standing eyes closed condition, not all the SCI participants were able to complete the allotted 2-min counting time. Therefore, for this condition, the first $60 \mathrm{~s}$ of counting were used for the analysis.

\section{Table 1 Experimental conditions}

\begin{tabular}{llll}
\hline Visual condition & Single task (count) & Single task (stand) & Dual task \\
\hline Eyes open & Sit and count & Stand & Stand and count \\
Eyes closed & NA & Stand & Stand and count \\
\hline
\end{tabular}

Abbreviation: NA, not applicable.
Signals from the force plates were processed offline using a dual-pass Butterworth filter with a low pass cut off frequency of $5 \mathrm{~Hz}$ to smooth the data and calculate center of pressure $(\mathrm{CoP})$ during each trial using custom-written routines in Matlab (Mathworks, Natick, MA, USA). Root mean squared and mean velocity of the $\mathrm{CoP}$ in the mediolateral and anteroposterior directions were calculated. No CoP measures were analyzed if the trial lasted less than $60 \mathrm{~s}$ since important low-frequency components of the signal may be missed. ${ }^{17,18}$

The tendency to switch from automatic to conscious control of movement was measured by asking subjects to complete the Movement Specific Reinvestment Scale (MSRS) ${ }^{12}$ after each single-task standing trial during eyes open and eyes closed conditions. The MSRS consists of 10 items that relate to concerns about movement self-consciousness, and conscious motor processing. We modified the questionnaire to reflect standing balance state-specific changes by substituting the words 'movement' with 'standing'. Increased reinvestment corresponds to a higher score out of a total possible score of 36 .

Subjective mental workload was assessed after each trial using the online National Aeronautics and Space Administration Task Load Index (NASATLX). ${ }^{19}$ An overall workload score was calculated based on a weighted average of six subscales. Scores range from 0 (least) to 100 (highest amount of workload). ${ }^{19}$

Although postural control is normally attributed to physiological factors, psychological perceptual factors such as fear of falling and balance selfconfidence have also been attributed to postural control changes. ${ }^{20,21}$ Balance self-confidence was assessed prior to each standing trial, while fear was assessed after each trial on a scale of $0-100 \%$ (0 least confident/fearful, 100 most confident/fearful). ${ }^{21}$

To assess the effects of the postural and suprapostural conditions during dual tasking, a relative measure of change, the dual-task cost (DTC), ${ }^{22,23}$ was calculated for all primary measures (except MSRS):

$$
\text { DTC }=\frac{\text { [dual task performance }- \text { single task performance] }}{\text { single task performance }} \times 100
$$

For our outcome measures, with the exception of TOT, a positive value represents a decrease in performance under the dual-task condition, whereas a negative value corresponds to an improvement in performance in the dual-task condition.

\section{Statistical analysis}

All data were analyzed using SPSS (v.21) statistical software (SPSS Inc, Chicago, IL, USA). The critical value for significance for all statistical tests was set at an alpha value of 0.05 . Reported values are mean \pm s.d. The mean difference between groups was calculated with $95 \%$ confidence interval (CI). Subject characteristics were summarized using descriptive statistics, and differences between groups were assessed using the independent $t$-test.

\section{Primary outcomes}

A two-way repeated measures analysis of variance (group: $\mathrm{SCI}, \mathrm{AB} \times$ task: single-task, dual-task) was performed on the primary measures: STime, ER and TOT. Normality of distribution was checked using the Shapiro-Wilk test. When the sphericity assumption was violated (Mauchly's Test), a GreenhouseGeisser correction was used. Differences in standing performance between the two groups for MSRS was assessed using independent $t$-tests. Levene's test was used to assess equality of variances. A two-way repeated measures multivariate analysis of variance was performed on the CoP measures. A log transform was performed on the data that were not normal. We used the data for a $120 \mathrm{~s}$ bout for eyes open since all SCI subjects could stand for the maximum amount of time, and $60 \mathrm{~s}$ for eyes closed.

\section{Secondary outcomes}

Differences in standing performance between the two groups for fear, confidence, and NASA-TLX was assessed using independent $t$-tests.

\section{RESULTS}

\section{Subject characteristics}

Seventeen subjects (nine SCI and eight AB controls) participated in the study. Individual characteristics of the SCI subjects are presented in 
Table 2 Subject characteristics and clinical outcomes of individual SCI subjects $(n=9)$

\begin{tabular}{|c|c|c|c|c|c|c|c|c|}
\hline Subject & Age (years) & Sex & Height $(\mathrm{cm})$ & Weight (kg) & Injury level & INCSCI & Chronicity (years) & Berg balance score \\
\hline 1 & 37 & M & 180 & 88 & c6-7 & C & 21 & 29 \\
\hline 2 & 33 & M & 175 & 70 & C5-6 & C & 11 & 10 \\
\hline 3 & 59 & $\mathrm{~F}$ & 155 & 59 & L4-5 & $\mathrm{D}$ & 2.5 & 52 \\
\hline 4 & 59 & M & 175 & 86 & C5-6 & C & 6 & NT \\
\hline 5 & 63 & $\mathrm{M}$ & 178 & 80 & C4-5 & $\mathrm{D}$ & 2 & 50 \\
\hline 6 & 51 & $\mathrm{M}$ & 170 & 75 & T3 & C & 12 & 35 \\
\hline 7 & 56 & $\mathrm{M}$ & 175 & 84 & C3-5 & $\mathrm{D}$ & 38 & 52 \\
\hline 8 & 64 & $\mathrm{M}$ & 173 & 82 & T12 & D & 1 & 55 \\
\hline 9 & 44 & $\mathrm{M}$ & 188 & 79 & $\mathrm{C} 1-2$ & C & 12 & 32 \\
\hline
\end{tabular}

Abbreviations: INCSCI, International Standards for Neurological Classification of Spinal Cord Injury; NT, not tested; SCl, spinal cord injury.

Table 2. Parametric tests revealed no statistically significant difference between the two groups (mean \pm s.d.) for age (SCI: $51.8 \pm 11.3$; AB: $49 \pm 11.3$ years), height (SCI: $174.3 \pm 8.9 ; \mathrm{AB} 177.6 \pm 10.4 \mathrm{~cm}$ ) or weight (SCI: $78.1 \pm 9.0 ; \quad \mathrm{AB}: \quad 78.5 \pm 13.6 \mathrm{~kg}$ ), $\quad P>0.05$ for all comparisons.

\section{Primary outcomes}

Standing time. All SCI subjects were able to stand for the maximum trial duration of 2 min during the eyes open condition for both singleand dual-task conditions. However, for total STime with eyes closed, significant interactions between task and group were observed $\left(F_{1,15}=5.4, P=0.034\right.$, partial $\left.\eta^{2}=0.266\right)$. On average, SCI subjects stood for $26 \%$ shorter duration in the dual-task with respect to the single-task condition compared to $\mathrm{AB}$ controls (Figure 1), with two of the SCI subjects unable to stand at all with eyes closed. The mean difference $(95 \% \mathrm{CI})$ between SCI and AB controls for the DTC of STime was $-26.15 \%(-45.54$ to $-6.76 \%)$.

Math task. Four of the nine SCI subjects could not complete the dual-task condition of standing and counting with eyes closed for the allotted minimum $60 \mathrm{~s}$ of STime, therefore their data could not be included in eyes closed math-task performance analysis. There were no statistically significant main or interaction effects in math-task

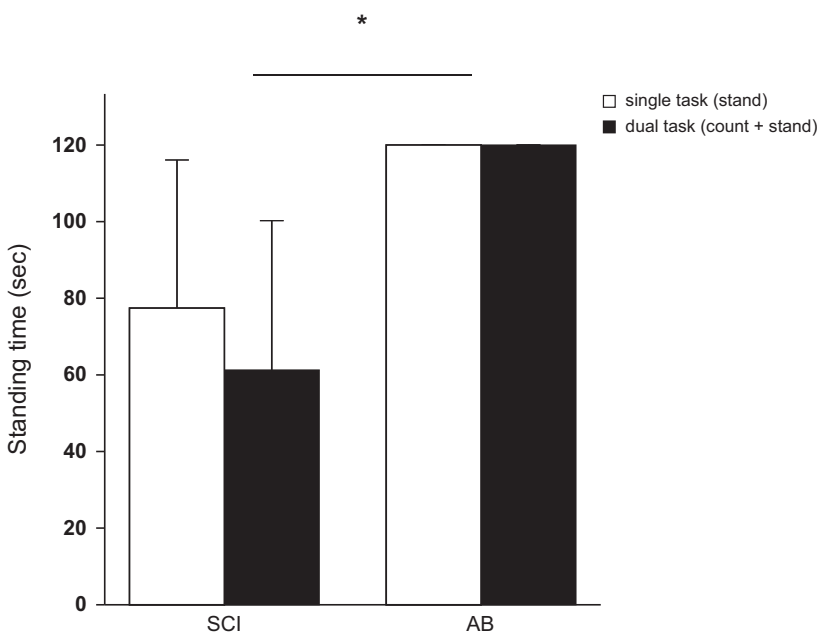

Figure 1 Standing time eyes closed between $\mathrm{SCl}$ and $\mathrm{AB}$ groups. Comparison between $\mathrm{SCl}$ and $\mathrm{AB}$ groups for the single task of standing and the dual task of standing and counting with eyes closed. Total standing duration was shorter among the $\mathrm{SCl}$ subjects in the dual-task condition compared to the $\mathrm{AB}$ controls $(P<0.05)$. Error bars represent $95 \%$ confidence interval. performance (ER or TOT) between the SCI and $\mathrm{AB}$ group, and between single- and dual-task conditions for either eyes open or eyes closed. The mean difference (95\% CI) for eyes open ER was $5.49 \%$ ( -8.98 to $19.96 \%)$, and TOT was $-15.41 \%$ ( -98.76 to 67.94$)$. The mean difference for eyes closed ER was $7.76 \%(-11.94$ to $27.46 \%)$ and TOT was -72.57 ( -322.49 to $177.35 \%)$. (Figure 2).

Movement reinvestment. SCI subjects reinvested significantly more conscious attention to postural control compared to $\mathrm{AB}$ subjects when standing quietly in both vision conditions (eyes open: $t_{15}=7.33$, $P<0.001$ ); (eyes closed: $t_{13}=6.86, P<0.001$ ) (Figure 3 ).

Center of pressure. Figure 4 illustrates representative single-subject data of CoP displacement plots during single-task (standing) and dualtask (standing and counting) from an SCI and $\mathrm{AB}$ participant. For the $\mathrm{CoP}$ measures, we observed a statistically significant multivariate main effect of group in both vision conditions (eyes open: Wilks' $\lambda=0.24$, $F_{4,12}=9.4, P=0.001$, partial $\eta^{2}=0.758$; eyes closed: Wilks' $\lambda=0.17$, $F_{4,9}=11.4, P=0.001$, partial $\left.\eta^{2}=0.835\right)$, and task for eyes closed (Wilks' $\lambda=0.34, F_{4,9}=4.3, P=0.032$, partial $\eta^{2}=0.658$ ). Significant univariate main effects for group and task were obtained for all CoP measures for eyes open. For eyes closed, significant univariate main effects for group and task for Root mean squared-mediolateral were observed. Generally, SCI subjects' mean velocity and sway area in anteroposterior and mediolateral directions were higher than those of controls in both vision conditions (Table 3 ).

Subjective mental workload. The subjective mental workload (NASATLX) of the SCI subjects during standing was significantly higher compared to $\mathrm{AB}$ controls (eyes open: $t_{10}=3.56, P=0.005$; CI $95 \%$ (11.27, 47.42); (eyes closed: $t_{13}=3.67, P=0.003$; CI 95\% (12.95, $50.18)$. The mean difference $(95 \%$ CI $)$ was $-29.34 \%(-47.57$ to $-11.11 \%)$ for eyes open and $-31.53 \%(-49.33$ to $-13.73 \%)$ for eyes closed.

Fear and confidence. We observed statistically significant differences between groups for fear $\left(\mathrm{t}_{6}=2.98, P=0.024\right.$, CI 95\% $\left.(6.20,60.87)\right)$ and confidence $\left(t_{6}=-4.58, P=0.003\right.$, CI 95\% $\left.(-65.67,-20.20)\right)$ in the eyes closed condition only. SCI subjects were $34 \%$ more fearful (mean difference $-33.54 \%(-56.03$ to $-11.05 \%)$ and $43 \%$ less confident (mean difference $42.93 \%$ (24.18-61.68\%) than AB controls during quiet standing with their eyes closed.

\section{DISCUSSION}

We found a significant DTC in total STime with eyes closed. We did not observe a significant dual-task effect on math-task performance, but we did find that SCI subjects had larger sway velocities and amplitudes and reinvested significantly more conscious control to 
a Eyes open

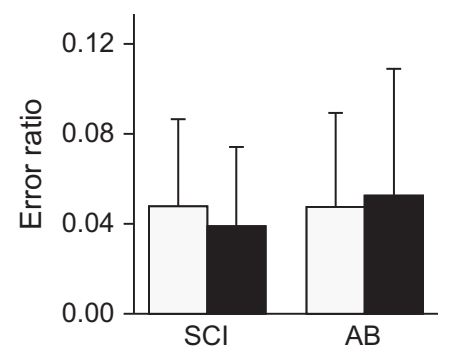

b Eyes closed

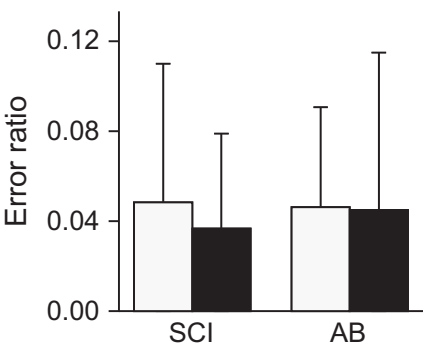

single task (count + sit)

dual task (count + stand)
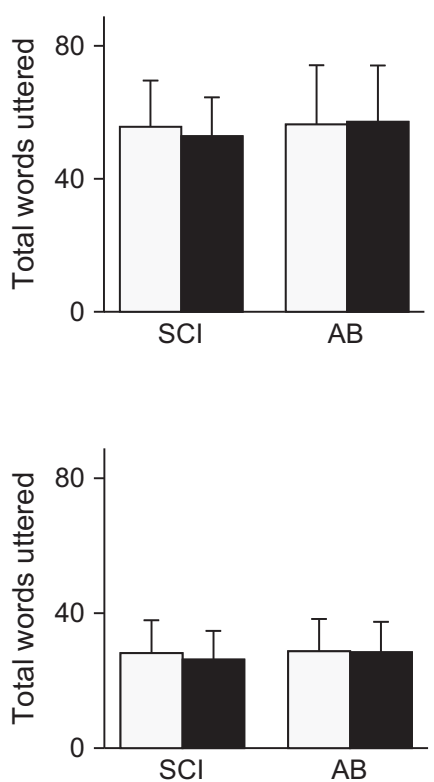

Figure 2 Math task performance between $\mathrm{SCl}$ and $\mathrm{AB}$ groups. Comparison between math-task performance (error ratio (left) and total words uttered (right)) during the single task of count and sit (white bars) and the dual task of count and stand (gray bars) for (a) eyes open, counting for $120 \mathrm{~s}$ and (b) eyes closed, counting for $60 \mathrm{~s}$. The $\mathrm{SCl}$ group performed equally as well as the $\mathrm{AB}$ group in all conditions $(P>0.05)$. Error bars represent $95 \%$ confidence interval.

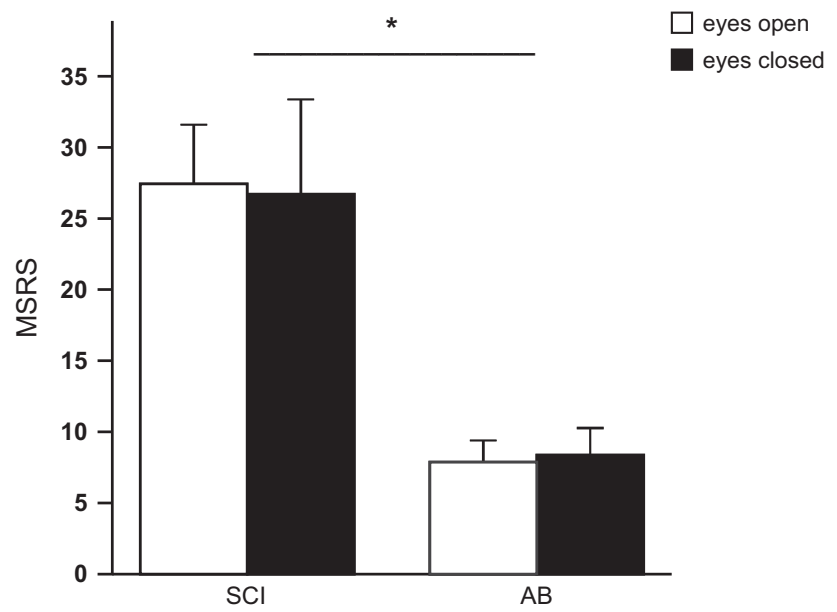

Figure 3 Movement reinvestment between $\mathrm{SCl}$ and $\mathrm{AB}$ groups. Comparison between $\mathrm{SCl}$ and $\mathrm{AB}$ groups for the amount of conscious control of standing posture (single task) during eyes open and eyes closed conditions. SCl subjects reinvested more conscious control to posture than $A B$ subjects in both vision conditions $(P<0.05)$. Higher MSRS scores indicate higher reinvestment. Error bars represent 95\% confidence interval.

posture than $\mathrm{AB}$ controls. These results appear to correspond to the higher ratings for fear, lower confidence and higher subjective workload in people with SCI during standing. However, even though we attempted to limit confounding variables by matching the groups, our small sample size limits us from confirming that the observed differences can be completely attributed to the SCI itself. Nevertheless, this data provide some insights into the attentional requirements of standing in this population and some considerations for understanding the recovery of postural control following SCI.
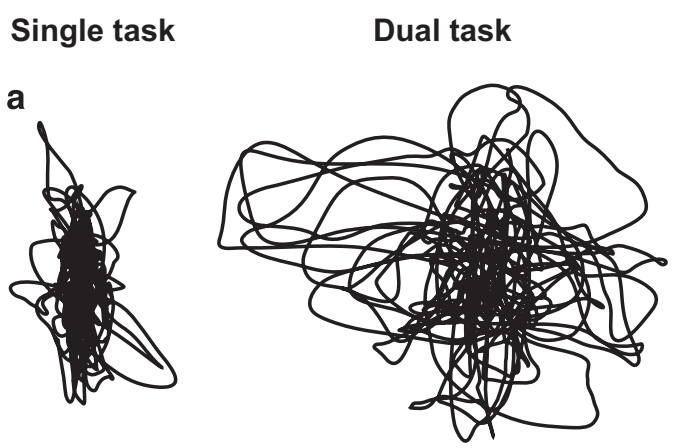

b

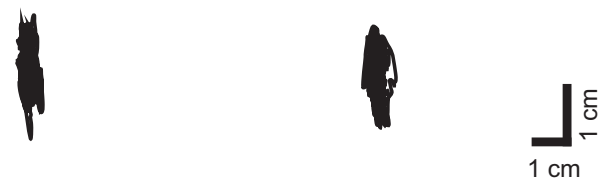

Figure $4 \mathrm{CoP}$ displacement for an $\mathrm{SCl}$ and $\mathrm{AB}$ subject. Representative $\mathrm{CoP}$ path plots for the single task of standing (left) and the dual task of standing and counting (right) from (a) $\mathrm{SCl}$ subject 5 and (b) an age-matched $\mathrm{AB}$ subject.

SCI subjects require greater attention to postural control than $\mathrm{AB}$ subjects in quiet standing

Standing balance in people with SCI may be compromised due to various levels of sensory loss, muscle weakness and spasticity, ${ }^{24}$ which could lead to greater movement reinvestment and postural sway. Our results are consistent with a study that compared the mean velocity and amplitude between SCI and $\mathrm{AB}$ controls $^{24}$ and a study that compared the amount of conscious reinvestment of stroke subjects with matched controls. ${ }^{25}$, Increased conscious control to posture could 


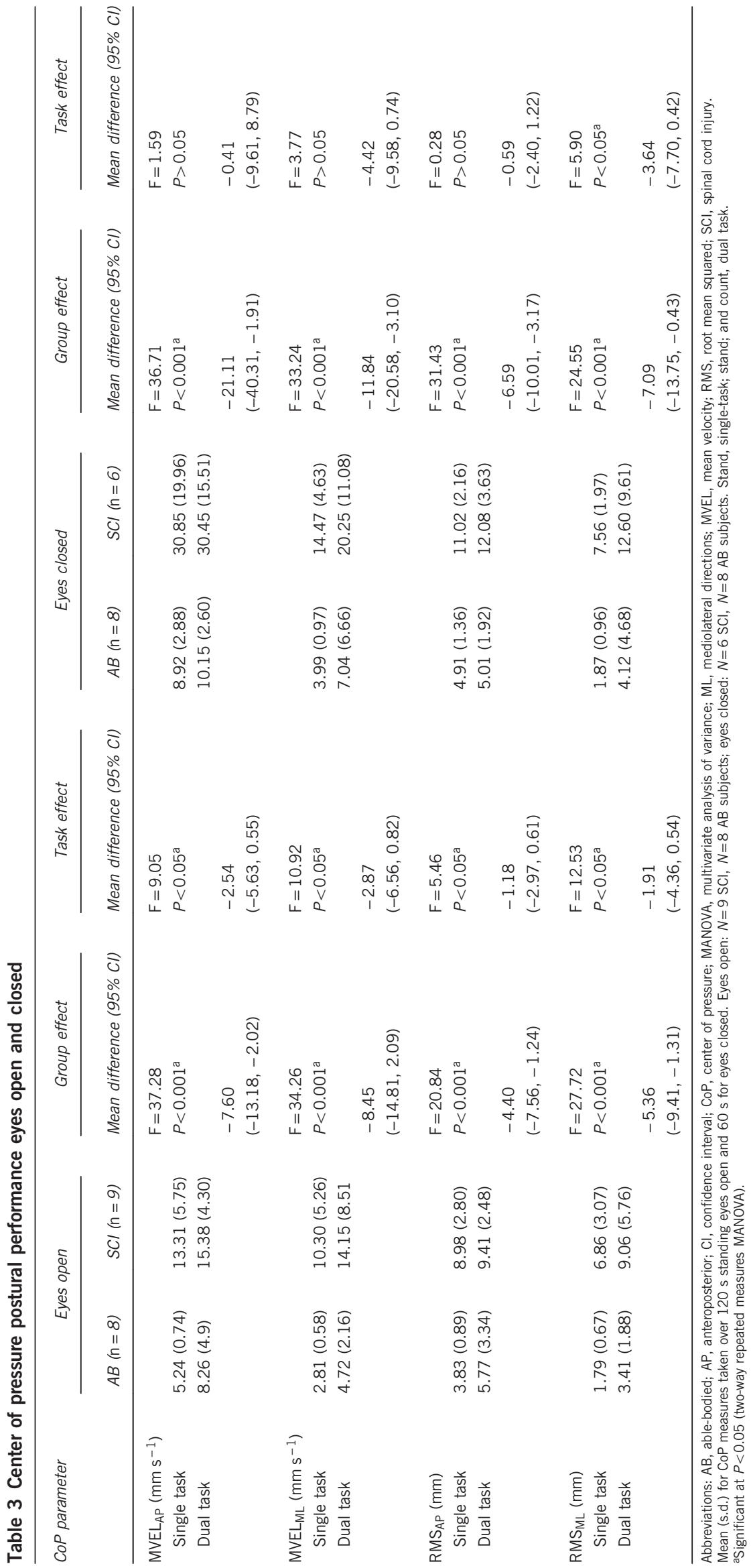


be viewed as an adaptive strategy by people with reduced postural control in order to maintain quiet standing.

Perceptual measures may influence the amount of resources devoted to processing tasks with regards to postural control. Psychological factors such as increased fear and decreased confidence have been reported to be associated with increased reinvestment. ${ }^{21}$ Our SCI subjects found standing more effortful and they were significantly more fearful and less confident than our $\mathrm{AB}$ controls, which could explain why we observed significant group effects between $\mathrm{SCI}$ and $\mathrm{AB}$ subjects for movement reinvestment and sway for both vision conditions.

\section{Dual task effect for standing time only}

We detected a dual-task effect for STime but not for the math task or postural sway and this could be explained in a number of ways. First, our SCI cohort was relatively high functioning. All could stand for at least 2 min with their eyes open and 7/9 participants could stand for at least $60 \mathrm{~s}$ with eyes closed. In addition, the median Berg Balance Scale score for our SCI group was 43/56, indicating that they would be considered as low falls risk. ${ }^{26}$

Second, the math task might not have been difficult enough. However, there have been many studies that have reported a dual-task effect when combining a backwards counting task with quiet standing. ${ }^{14,16}$ It is possible that our lack of significant DT effects on math performance and CoP parameters may have been hampered by our attempt to normalize the timeframe by only selecting those subjects who could stand for at least $60 \mathrm{~s}$, thereby inadvertently limiting our SCI group to those who were high functioning. This is underscored by the fact that our STime results, which included the two subjects who could not stand at all with their eyes closed, revealed a significant DTC between our SCI and AB groups. Therefore, our protocol revealed that the math task may not have been challenging enough for subjects who could stand for at least $60 \mathrm{~s}$ eyes closed, yet was too difficult for those who could not stand for the minimum $60 \mathrm{~s}$.

A key limitation in this study was the small sample size and use of multiple outcomes and comparisons. Moreover, with the exception of STime with eyes closed, the differences in DTC between groups of our primary performance measures were small, with large amounts of variability, consistent with the idea that our postural and secondary tasks may not have been challenging enough. Consideration must also be made when involving verbal responses, since depending on the level of the lesion, SCI can lead to impairment of the respiratory system ${ }^{27}$ and changes in respiration and speech have been shown to have an effect on postural control. ${ }^{28}$ Our SCI group was living and working in the community and physically active, and so generalizability of our findings may be limited to only the higher-functioning strata of the SCI population.

\section{Clinical message}

Adding a suprapostural task to balance training may help reveal the attentional resources devoted to postural control in people with SCI, but the dual task must be sufficiently difficult to challenge balance especially in high-functioning people with SCI.

\section{CONCLUSIONS}

Our study has demonstrated that total STime during eyes closed is adversely affected with the addition of a math task. Threat-related factors, such as increased fear, decreased confidence and increased perceived mental workload were observed in parallel with increases in postural sway and conscious control of standing in subjects with SCI. However, it appears that for people with SCI with good balance function, standing on a firm surface with eyes open and performing a concurrent suprapostural task of minimal mental workload would not greatly affect their postural control any more than the single task of standing quietly.

\section{CONFLICT OF INTEREST}

The authors declare no conflict of interest.

\section{ACKNOWLEDGEMENTS}

We thank Franco Chan for his valuable technical support during the study.

1 Woollacott M, Shumway-Cook A. Attention and the control of posture and gait: a review of an emerging area of research. Gait Posture 2002; 16: 1-14.

2 Maylor EA, Allison S, Wing AM. Effects of spatial and nonspatial cognitive activity on postural stability. Br J Psychol 2001; 92: 319-338.

3 Maki BE, Mcllroy WE. Cognitive demands and cortical control of human balancerecovery reactions. J Neural Transm 2007; 114: 1279-1296.

4 Maki BE, Zecevic A, Bateni H, Kirshenbaum N, Mcllroy WE. Cognitive demands of executing postural reactions: does aging impede attention switching? Neuroreport 2001; 12: 3583-3587.

5 Andersson G, Hagman J, Talianzadeh R, Svedberg A, Larsen HC. Dual-task study of cognitive and postural interference in patients with vestibular disorders. Otol Neurotol 2003; 24: 289-293.

6 Boes MK, Sosnoff JJ, Socie MJ, Sandroff BM, Pula JH, Motl RW. Postural control in multiple sclerosis: effects of disability status and dual task. J Neurol Sci 2012; 315: 44-48.

7 Bourlon C, Lehenaff L, Batifoulier C, Bordier A, Chatenet A, Desailly E et al. Dual-tasking postural control in patients with right brain damage. Gait Posture 2014; 39: 188-193.

8 Dromey C, Jarvis E, Sondrup S, Nissen S, Foreman KB, Dibble LE. Bidirectional interference between speech and postural stability in individuals with Parkinson's disease. Int J Speech Lang Pathol 2010; 12: 446-454.

9 Lee GE, Bae H, Yoon TS, Kim JS, Yi TI, Park JS. Factors that influence quiet standing balance of patients with incomplete cervical spinal cord injuries. Ann Rehabil Med 2012; 36: 530-537.

10 Lemay J, Gagnon D, Duclos C, Gauthier C, Nadeau S. Influence of visual inputs on quasi-static standing postural steadiness in individuals with spinal cord injury. Gait Posture 2013; 38: 357-360.

11 Remaud A, Boyas S, Lajoie Y, Bilodeau M. Attentional focus influences postural control and reaction time performances only during challenging dual-task conditions in healthy young adults. Exp Brain Res 2013; 231: 219-229.

12 Masters R, Maxwell J. The theory of reinvestment. Int Rev Sport Exerc Psychol 2008; 1: $160-183$.

13 Beilock SL, Carr TH, MacMahon C, Starkes JL. When paying attention becomes counterproductive: impact of divided versus skill-focused attention on novice and experienced performance of sensorimotor skills. J Exp Psychol Appl 2002; 8: 6-16.

14 Swanenburg J, de Bruin ED, Uebelhart D, Mulder T. Compromising postural balance in the elderly. Gerontology 2009; 55: 353-360.

15 Van Daele U, Hagman F, Truijen S, Vorlat P, Van Gheluwe B, Vaes P. Decrease in postural sway and trunk stiffness during cognitive dual-task in nonspecific chronic low back pain patients, performance compared to healthy control subjects. Spine 2010; 35: 583-589.

16 Pellecchia GL. Postural sway increases with attentional demands of concurrent cognitive task. Gait Posture 2003; 18: 29-34.

17 Carpenter MG, Frank JS, Winter DA, Peysar GW. Sampling duration effects on centre of pressure summary measures. Gait Posture 2001; 13: 35-40.

18 van der Kooij H, Campbell AD, Carpenter MG. Sampling duration effects on centre of pressure descriptive measures. Gait Posture 2011; 34: 19-24.

19 Hart SG, Staveland LE. Development of NASA-TLX (Task Load Index): results of empirical and theoretical research. Adv Psychol 1988; 52: 139-183.

20 Carpenter MG. Postural, physiological and psychological reactions to challenging balance: does age make a difference? Age Ageing 2006; 35: 298-303.

21 Huffman JL, Horslen BC, Carpenter MG, Adkin AL. Does increased postural threat lead to more conscious control of posture? Gait Posture 2009; 30: 528-532.

22 Siu K-C, Woollacott MH. Attentional demands of postural control: the ability to selectively allocate information-processing resources. Gait Posture 2007; 25: 121-126.

23 McCulloch K. Attention and dual-task conditions: physical therapy implications for individuals with acquired brain injury. J Neurol Phys Ther 2007; 31: 104-118.

24 Lemay J-F, Gagnon D, Duclos C, Grangeon M, Gauthier C, Nadeau S. Influence of visual inputs on quasi-static standing postural steadiness in individuals with spinal cord injury. Gait Posture 2013; 38: 357-360.

25 Orrell AJ, Masters RSW, Eves FF. Reinvestment and movement disruption following stroke. Neurorehabil Neural Repair 2009; 23: 177-183.

26 Stevenson TJ. Detecting change in patients with stroke using the Berg Balance Scale. Aust J Physiother 2001; 47: 29-38.

27 Winslow C, Rozovsky J. Effect of spinal cord injury on the respiratory system. Am J Phys Med Rehabil 2003; 82: 803-814.

28 Bouisset S, Duchêne J-L. Is body balance more perturbed by respiration in seating than in standing posture? Neuroreport 1994; 5: 957. 